\title{
A Modified Bayesian Filter for Randomly Delayed Measurements
}

\author{
Abhinoy Kumar Singh, Paresh Date and Shovan Bhaumik
}

\begin{abstract}
The traditional Bayesian approximation framework for filtering in discrete time systems assumes that the measurement is available at every time instant. But in practice, the measurements could be randomly delayed. In the literature, the problem has been examined and solution is provided by restricting the maximum number of delay to one or two time steps. This paper develops an approach to deal with the filtering problems with an arbitrary number of delays in measurement. Pursuing this objective, traditional Bayesian approximation to nonlinear filtering problem is modified by reformulating the expressions of mean and covariances which appear during the measurement update. We use the cubature quadrature rule to evaluate the multivariate integral expressions for the mean vector and the covariance matrix which appear in the developed filtering algorithm. We compare the new algorithm which accounts for delay with the existing CQKF heuristics on two different examples and demonstrate how accounting for a random delay improves the filtering performance.
\end{abstract}

Index Terms-Nonlinear filtering, Bayesian framework of filtering, Random delay in measurements, Cubature quadrature Kalman filter.

\section{INTRODUCTION}

$\mathbf{T}$ HERE are several efficient filtering techniques available for nonlinear systems in the literature. Some of the more popular techniques include the particle filter (PF) [1], the extended Kalman filter (EKF) [2], the unscented Kalman filter (UKF) [3], the Gauss-Hermite filter (GHF) [4], the central difference filter (CDF) [5], the cubature Kalman filter (CKF) [6] etc. Out of these approximations, CKF is quite popular, especially in the field of tracking, due to its high accuracy and computational efficiency. Very recently, the CKF was generalized and cubature quadrature Kalman filter (CQKF) [7] was introduced for achieving better accuracy.

The traditional framework of filtering is developed with the assumption that the current measurement is available at every time instant. But, in practice it is not always. The estimator may receive the measurements with a random delay in time. For example, the measurement received at the time instant ' $t$ ' may actually belongs to any time ' $t-\tau$ ' where $0 \leq \tau<t$. The equality sign represents that the measurement may be non-delayed and belongs to the current time step itself.

A. K. Singh is with the Department of Electrical Engineering, Indian Institute of Technology Patna, Bihar 800013, India(e-mail:abhinoy@iitp.ac.in)

P. Date is with the Department of Mathematics, College of Engineering, Design and Physical Sciences at Brunel University London Uxbridge, UB8 3PH, United Kingdom(phone:+44 1895265613 ; fax: +44 1895269727 ; e-mail:paresh.date@brunel.ac.uk)

S. Bhaumik is with the Department of Electrical Engineering, Indian Institute of Technology Patna, Bihar 800013, India(phone:+91 6122552049 fax: 91-612-2277383; e-mail:shovan.bhaumik@iitp.ac.in)
This delay may occur before arrival of measurement to the sensor or during its propagation from sensor to estimator due to the limited bandwidth. In practice, this kind of problems usually occur in aerospace and underwater target tracking [8], communication [9], control applications [10] etc.

In the filtering literature, extensive research has been carried out for filtering of linear systems with delay in measurements. But focusing on the nonlinear systems, we find very few noticeable papers addressing the problems of random delays. Hermoso-Carazo et. al. introduced a nonlinear filtering algorithm for one-time step [11] and two-time step [12] randomly delayed measurements using the extended and the unscented Kalman filter approach. The work has been extended for correlated noises in [14]. Later, Wang et. al. [13] incorporated cubature Kalman filter (CKF) [6] to solve the nonlinear filtering problems with one-step randomly delayed measurement. All these works are restricted to delays extending to one or two time steps.

In this paper, the conventional Bayesian framework of filtering using numerical integration is modified to incorporate any arbitrary number of random delays in measurements. We reformulate the standard multivariate integral expressions under Gaussian approximation to account for delays. Like the conventional CKF/CQKF algorithms, the modified algorithm includes intractable integrals for nonlinear systems, which are approximated numerically.

The authors choose to implement the cubature quadrature approach, recently introduced in CQKF [7], to solve the intractable integrals appeared in the proposed algorithm with delayed measurements. The CQKF under modified framework is named as the randomly delayed cubature quadrature Kalman filter (CQKF-RD). From the simulation results, it could be justified that the CQKF-RD performs better than the ordinary CQKF for filtering with delayed measurements.

\section{Modified Approximate Filtering FOR RANDOMLY Delayed Measurements}

Let us consider a dynamic system whose state space model could be described in terms of the following equations:

$$
\begin{gathered}
\text { State equation } \quad \mathbf{x}_{k}=\phi_{k-1}\left(\mathbf{x}_{k-1}, k-1\right)+\mathbf{q}_{k-1}, \\
\text { Measurement equation } \quad \mathbf{z}_{k}=\gamma_{k}\left(\mathbf{x}_{k}, k\right)+\mathbf{v}_{k}
\end{gathered}
$$

where $\mathbf{x}_{k} \in \Re^{n}$ denotes the state vector of a system and $\mathbf{z}_{k} \in \Re^{d}$ is the measurement at any instant $k$ where $k \in$ $\{0,1,2,3, \ldots, N\} . \phi_{k}\left(\mathbf{x}_{k}, k\right)$ and $\gamma_{k}\left(\mathbf{x}_{k}, k\right)$ are known nonlinear functions of $\mathbf{x}_{k}$ and $k$. The process noise $\mathbf{q}_{k} \in \Re^{n}$ and 
measurement noise $\mathbf{v}_{k} \in \Re^{d}$ are assumed to be uncorrelated, white and distributed normally with covariance $\mathbf{Q}_{k}$ and $\mathbf{R}_{k}$ respectively.

As discussed earlier, the assumption that the current measurement is received at each time step does not always hold true in practice. It may belong to the same time step or may be a randomly delayed measurement from a previous time step. To address this situation, we need to modify the measurement equation of the dynamic state space model.

To modify the measurement model in a tractable fashion, we need to fix the maximum number of delays. Let us assume this number as $(\mathrm{N}-1)$ time steps. The value of $\mathrm{N}$ can be chosen by the practitioner, subject to the following considerations:

- If $\mathrm{N}$ is chosen very small as compared to the actual delay possible, then no measurement will appear at several time steps. It may result in loss of data and subsequently, poor filtering accuracy.

- As will be seen in the next section, the complexity of filtering rises with increasing $\mathrm{N}$.

For $(\mathrm{N}-1)$-delayed systems, at $k^{\text {th }}$ time step either any measurement $\mathbf{z}_{k-i}(0 \leq i \leq \mathrm{N}-1)$ arrives or no measurement arrives. If no measurement arrives, the measurement data stored in the sensor is not updated and hence the estimator receives the measurement received at previous time step itself.

To model the delayed measurements at $k^{t h}$ time instant, corresponding to each $(k-i)^{t h}(0 \leq i \leq \mathrm{N}-1)$ time step we need a random number which takes a value either 1 or 0 . The $k^{\text {th }}$ step measurement actually belongs to a step for which this value is 1 . To this regard, let $\beta_{j}(j=1,2, \ldots, \mathrm{N})$ be the independent Bernoulli random variables taking values either 0 or 1 with probability $P\left(\beta_{j}=1\right)=p=E\left[\beta_{j}\right]$, and $P\left(\beta_{j}=0\right)=1-p$. If $\mathbf{y}_{k}$ represents the measurement received at $k^{\text {th }}$ time instant, then

$$
\begin{aligned}
& \mathbf{y}_{k}=\left(1-\beta_{1}\right) \mathbf{z}_{k}+\beta_{1}\left(1-\beta_{2}\right) \mathbf{z}_{k-1}+\beta_{1} \beta_{2}\left(1-\beta_{3}\right) \mathbf{z}_{k-2}+\ldots \\
& +\left(\prod_{i=1}^{\mathrm{N}-1} \beta_{i}\right)\left(1-\beta_{\mathrm{N}}\right) \mathbf{z}_{(k-\mathrm{N}+1)}+\left[1-\left(1-\beta_{1}\right)-\beta_{1}\left(1-\beta_{2}\right)\right. \\
& \left.-\beta_{1} \beta_{2}\left(1-\beta_{3}\right)-\ldots-\left(\prod_{i=1}^{\mathrm{N}-1} \beta_{i}\right)\left(1-\beta_{\mathrm{N}}\right)\right] \mathbf{y}_{k-1} \\
& =\beta^{(j, 0)} \mathbf{z}_{k}+\beta^{(j, 1)} \mathbf{z}_{k-1}+\beta^{(j, 2)} \mathbf{z}_{k-2}+\ldots+\beta^{(j, \mathrm{~N}-1)} \mathbf{z}_{(k-\mathrm{N}+1)} \\
& +\left(1-\sum_{i=0}^{\mathrm{N}-1} \beta^{(j, i)}\right) \mathbf{y}_{k-1},
\end{aligned}
$$

where $\beta_{0}=1$ and $\beta^{(j, i)}=\left(\prod_{j=0}^{i} \beta_{j}\right)\left(1-\beta_{i+1}\right)$. From the above expression, it is clear that the $\mathbf{y}_{k}$ is $i$ step delayed if $\beta_{i+1}=0$ and $\beta_{j}=1 \forall j \neq i$.

$$
\text { Hence, } \mathbf{y}_{k}=\sum_{i=0}^{\mathrm{N}-1}\left(\beta^{(j, i)} \mathbf{z}_{k-i}\right)+\left(1-\sum_{i=0}^{\mathrm{N}-1} \beta^{(j, i)}\right) \mathbf{y}_{k-1} \text {. }
$$

This is the modified measurement model which actually appears in case the measurement is randomly delayed in time. The values of $\beta^{(j, i)}$ will be either 0 or 1 . At any specific instant, at most one $\beta^{(j, i)}(\forall 0 \leq i \leq \mathrm{N}-1)$ can be 1 while all the remaining will be 0 . If $\beta^{(j, i)}=1$ and $\beta^{(j, k)}=0$ $(\forall \quad k \neq i)$, then the received measurement will be $i^{t h}$ time step delayed measurement. If all the $\beta^{(j, i)}(\forall 0 \leq i \leq \mathrm{N}-1)$ are zero, it represents that no measurement is received at the corresponding time instant. In such case $\mathbf{y}_{k-1}$ will propagate at $k^{\text {th }}$ instant i.e. $\mathbf{y}_{k}=\mathbf{y}_{k-1}$. It can be noticed in equation (4).

Lemma 1: The probability that the measurement received at $k^{\text {th }}$ time step is actually $i$ time step delayed, is $\mathrm{p}_{i}=p^{i}(1-p)$.

Proof: $\mathrm{p}_{i}=E\left[\beta^{(j, i)}\right]$.

As $\beta_{j}$ is independent of $\beta_{k} \forall j \neq k$, hence

$$
\mathrm{p}_{i}=E\left[\beta^{(j, i)}\right]=E\left[\left(\prod_{j=0}^{i} \beta_{j}\right)\right] E\left[\left(1-\beta_{i+1}\right)\right] .
$$

Moreover, $E\left[\left(\prod_{j=0}^{i} \beta_{j}\right)\right]=\left(\prod_{j=0}^{i} E\left[\beta_{j}\right]\right)=p^{i}$,

$$
\text { and } \quad E\left[\left(1-\beta_{i+1}\right)\right]=(1-p) \text {. }
$$

Substituting equations (6) and (7) into (5), we get

$$
\mathrm{p}_{i}=E\left[\beta^{(j, i)}\right]=p^{i}(1-p)
$$

Lemma 2: The probability that no measurement will be received at $k^{\text {th }}$ time instant can be given as $\bar{p}_{k}=p^{\mathrm{N}}$.

Proof: $\bar{p}_{k}$ can be given as

$$
\bar{p}_{k}=E\left[1-\sum_{i=0}^{\mathrm{N}-1} \beta^{(j, i)}\right] \text {. }
$$

We can write

$$
E\left[1-\sum_{i=0}^{\mathrm{N}-1} \beta^{(j, i)}\right]=1-\sum_{i=0}^{\mathrm{N}-1} E\left[\beta^{(j, i)}\right]
$$

Substituting (8), we get

$$
\bar{p}_{k}=E\left[1-\sum_{i=0}^{N-1} \beta^{(j, i)}\right]=1-\sum_{i=0}^{N-1} p^{i}(1-p)=p^{N}
$$

Remark 1: As the value of $p$ is usually small relative to unity, the probability that no measurement arrives at $k^{\text {th }}$ time instant i.e. $\bar{p}_{k}=p^{\mathrm{N}}$ will be very small. Even for higher $p$, it can be make smaller by increasing the value of $\mathrm{N}$.

As the measurement model is modified, the mean and the covariance of measurement along with the cross-covariance which we denote as $\hat{\mathbf{z}}_{k}, \mathbf{P}_{k \mid k-1}^{\mathbf{z z}}$ and $\mathbf{P}_{k \mid k-1}^{\mathbf{x z}}$ respectively, should be modified in Bayesian framework of filtering. Hence, the problem reduces to reformulation of these expressions and deriving $\hat{\mathbf{y}}_{k}, \mathbf{P}_{k \mid k-1}^{\mathbf{y y}}$ and $\mathbf{P}_{k \mid k-1}^{\mathbf{x y}}$ for modified model.

The proposed modification is introduced in two steps. In the first step, the reformulation is done under the assumption that no measurement is lost for $(\mathrm{N}-1)$-delayed filter. In the second step, these expressions are further modified and the possibility that some measurements may be lost is incorporated.

The purpose of performing this modification in two steps is simple. The chances of the measurement being delayed 
by a large number of sample times is low, so that the value of $\mathrm{p}$ is small. Consequently, the probability of not receiving any measurement, $\bar{p}_{k}$ will be very small and hence it can be ignored. Subsequently, the filtering can be performed with the expressions derived in the first step only in order to reduce the computational complexity. But if this is not the case, the expression derived in the second step can be employed.

\section{A. Step 1: If no measurement is lost for $(N-1)$-delay}

Say, the measurement at $k^{t h}$ time instant under the condition that no measurement is lost for $(\mathrm{N}-1)$-delay is $\mathbf{Y}_{k}$, then

$$
\mathbf{Y}_{k}=\sum_{i=0}^{\mathrm{N}-1} \beta^{(j, i)} \mathbf{z}_{k-i}
$$

We derive the expressions for $\hat{\mathbf{Y}}_{k}, \mathbf{P}_{k \mid k-1}^{\mathbf{Y Y}}$ and $\mathbf{P}_{k \mid k-1}^{\mathbf{x Y}}$ in a series of lemmas next.

Lemma 3: If no measurement is lost for $(\mathrm{N}-1)$-delay, the estimate of measurement at $k^{t h}$ time instant can be given as

$$
\hat{\mathbf{Y}}_{k \mid k-1}=(1-p) \sum_{i=0}^{\mathrm{N}-1} p^{i} \hat{\mathbf{z}}_{k-i \mid k-1}
$$

Proof: From equation (11)

$\hat{\mathbf{Y}}_{k \mid k-1}=E\left[\mathbf{Y}_{k}\right]=E\left[\sum_{i=0}^{\mathrm{N}-1} \beta^{(j, i)} \mathbf{z}_{k-i}\right]=\sum_{i=0}^{\mathrm{N}-1} E\left[\beta^{(j, i)}\right] \hat{\mathbf{z}}_{k-i \mid k-1}$.

Substituting (8), $\quad \hat{\mathbf{Y}}_{k \mid k-1}=(1-p) \sum_{i=0}^{\mathrm{N}-1} p^{i} \hat{\mathbf{z}}_{k-i \mid k-1}$.

Lemma 4: If no measurement is lost for $(\mathrm{N}-1)$-delay, the covariance of measurement at $k^{\text {th }}$ time instant is

$$
\begin{gathered}
\mathbf{P}_{k \mid k-1}^{\mathbf{Y Y}}=(1-p) \sum_{i=0}^{\mathrm{N}-1} p^{i} \mathbf{P}_{k-i \mid k-1}^{\mathbf{z z}}+(1-p) \sum_{i=0}^{\mathrm{N}-1} p^{i} \\
\left(1-p^{i}(1-p)\right)\left(\left(\hat{\mathbf{z}}_{k-i \mid k-1}\right)\left(\hat{\mathbf{z}}_{k-i \mid k-1}\right)^{T}\right) .
\end{gathered}
$$

Proof: From equations (11) and (12), we get

$$
\begin{aligned}
& \mathbf{Y}_{k}-\hat{\mathbf{Y}}_{k \mid k-1}=\sum_{i=0}^{\mathrm{N}-1} \beta^{(j, i)} \mathbf{z}_{k-i}-(1-p) \sum_{i=0}^{\mathrm{N}-1} p^{i} \hat{\mathbf{z}}_{k-i \mid k-1} \\
& =\underbrace{\sum_{i=0}^{\mathrm{N}-1} \beta^{(j, i)}\left(\mathbf{z}_{k-i}-\hat{\mathbf{z}}_{k-i \mid k-1}\right)}_{\mathbf{M}_{1}}+\underbrace{\sum_{i=0}^{N-1}\left(\beta^{(j, i)}-(1-p) p^{i}\right) \hat{\mathbf{z}}_{k-i \mid k-1}}_{\mathbf{M}_{2}}
\end{aligned}
$$

$$
\mathbf{P}_{k \mid k-1}^{\mathbf{Y Y}}=E\left[\mathbf{M}_{1} \mathbf{M}_{1}^{T}\right]+E\left[\mathbf{M}_{1} \mathbf{M}_{2}^{T}\right]+E\left[\mathbf{M}_{2} \mathbf{M}_{1}^{T}\right]+E\left[\mathbf{M}_{2} \mathbf{M}_{2}^{T}\right] .
$$

$$
\begin{aligned}
E\left[\mathbf{M}_{1} \mathbf{M}_{1}^{T}\right] & =\sum_{i=0}^{\mathrm{N}-1} E\left[\beta^{(j, i)}\right]^{2} E\left[\left(\mathbf{z}_{k-i}-\hat{\mathbf{z}}_{k-i \mid k-1}\right)\right. \\
\left.\left(\mathbf{z}_{k-i}-\hat{\mathbf{z}}_{k-i \mid k-1}\right)^{T}\right] & =\sum_{i=0}^{\mathrm{N}-1} E\left[\beta^{(j, i)}\right] \mathbf{P}_{k-i \mid k-1}^{\mathbf{z z}} .
\end{aligned}
$$

From (8), $E\left[\mathbf{M}_{1} \mathbf{M}_{1}^{T}\right]=(1-p) \sum_{i=0}^{\mathrm{N}-1} p^{i} \mathbf{P}_{k-i \mid k-1}^{\mathbf{z z}}$
Next, $\quad E\left[\mathbf{M}_{1} \mathbf{M}_{2}^{T}\right]=\sum_{s=0}^{\mathrm{N}-1} \sum_{t=0}^{\mathrm{N}-1} E\left[\mathbf{A}_{s} \mathbf{A}_{t}^{T}\right]$

where $\mathbf{A}_{s}=\beta^{j, s}\left(\mathbf{z}_{k-s}-\hat{\mathbf{z}}_{k-s \mid k-1}\right)$

and, $\mathbf{A}_{t}=\left(\beta^{j, t}-(1-p) p^{t}\right) \hat{\mathbf{z}}_{k-t \mid k-1}$.

For any $s$ and $t$,

$$
\begin{gathered}
E\left[\mathbf{A}_{s} \mathbf{A}_{t}^{T}\right]=E\left[\beta^{j, s}\left(\beta^{j, t}-(1-p) p^{t}\right)\left(\left(\mathbf{z}_{k-s}\right)\left(\hat{\mathbf{z}}_{k-t \mid k-1}\right)^{T}\right)\right] \\
-E\left[\beta^{j, s}\left(\beta^{j, t}-(1-p) p^{t}\right)\left(\left(\hat{\mathbf{z}}_{k-s \mid k-1}\right)\left(\hat{\mathbf{z}}_{k-t \mid k-1}\right)^{T}\right)\right] \\
\text { or, } E\left[\mathbf{A}_{s} \mathbf{A}_{t}^{T}\right]=E\left[\beta^{j, s}\left(\beta^{j, t}-(1-p) p^{t}\right)\right. \\
\left.-\beta^{j, s}\left(\beta^{j, t}-(1-p) p^{t}\right)\right]\left(\left(\hat{\mathbf{z}}_{k-s \mid k-1}\right)\left(\hat{\mathbf{z}}_{k-t \mid k-1}\right)^{T}\right) \\
\text { or, } \quad E\left[\mathbf{A}_{s} \mathbf{A}_{t}^{T}\right]=0
\end{gathered}
$$

Substituting (18) into (17), $\quad E\left[\mathbf{M}_{1} \mathbf{M}_{2}^{T}\right]=0$.

Similarly, it can be shown that $E\left[\mathbf{M}_{2} \mathbf{M}_{1}^{T}\right]=0$.

$$
\begin{gathered}
E\left[\mathbf{M}_{2} \mathbf{M}_{2}^{T}\right]=\sum_{i=0}^{N-1} E\left[\left(\beta^{(j, i)}-(1-p) p^{i}\right)^{2}\right]\left(\hat{\mathbf{z}}_{k-i \mid k-1}\right) \times \\
\left(\hat{\mathbf{z}}_{k-i \mid k-1}\right)^{T}=\sum_{i=0}^{N-1} E\left[\left(\beta^{(j, i)}\right)^{2}+\left((1-p) p^{i}\right)^{2}\right. \\
\left.-2 \beta^{(j, i)}\left((1-p) p^{i}\right)\right]\left(\hat{\mathbf{z}}_{k-i \mid k-1}\right)\left(\hat{\mathbf{z}}_{k-i \mid k-1}\right)^{T} .
\end{gathered}
$$

In the above expression

$$
E\left[\left(\beta^{(j, i)}\right)^{2}\right]=E\left[\beta^{(j, i)}\right]=p^{i}(1-p) .
$$

hence, $E\left[\mathbf{M}_{2} \mathbf{M}_{2}^{T}\right]=\sum_{i=0}^{\mathrm{N}-1}\left(p^{i}(1-p)-p^{2 i}(1-p)^{2}\right) \times$

$$
\left(\left(\hat{\mathbf{z}}_{k-i \mid k-1}\right) \times\left(\hat{\mathbf{z}}_{k-i \mid k-1}\right)^{T}\right) .
$$

Substituting (16), (19), (20) and (21) into (15), we get

$$
\begin{gathered}
\mathbf{P}_{k \mid k-1}^{\mathbf{Y Y}}=(1-p) \sum_{i=0}^{\mathrm{N}-1} p^{i} \mathbf{P}_{k-i \mid k-1}^{\mathbf{z Z}}+(1-p) \sum_{i=0}^{\mathrm{N}-1} p^{i}(1- \\
\left.p^{i}(1-p)\right)\left(\left(\hat{\mathbf{z}}_{k-i \mid k-1}\right)\left(\hat{\mathbf{z}}_{k-i \mid k-1}\right)^{T}\right) .
\end{gathered}
$$

Lemma 5: If no measurement is lost for $(\mathrm{N}-1)$-delay, the cross-covariance between state and measurement at $k^{t h}$ time instant can be given as

$$
\mathbf{P}_{k \mid k-1}^{\mathbf{x Y}}=(1-p) \sum_{i=0}^{\mathrm{N}-1} p^{i} \mathbf{P}_{k-i \mid k-1}^{\mathbf{x z}} .
$$

Proof: From (14), $\mathbf{Y}_{k}-\hat{\mathbf{Y}}_{k \mid k-1}=\left(\mathbf{M}_{1}+\mathbf{M}_{2}\right)$. Hence,

$$
\mathbf{P}_{k \mid k-1}^{\mathbf{x Y}}=E\left[\left(\mathbf{x}_{k}-\hat{\mathbf{x}}_{k \mid k-1}\right)\left(\mathbf{M}_{1}+\mathbf{M}_{2}\right)^{T}\right] .
$$


Now, $E\left[\left(\mathbf{x}_{k}-\hat{\mathbf{x}}_{k \mid k-1}\right)\left(\mathbf{M}_{1}\right)^{T}\right]$

$$
\begin{aligned}
& =E\left[\left(\mathbf{x}_{k}-\hat{\mathbf{x}}_{k \mid k-1}\right) \times\left(\sum_{i=0}^{\mathrm{N}-1} \beta^{(j, i)}\left(\mathbf{z}_{k-i}-\hat{\mathbf{z}}_{k-i \mid k-1}\right)\right)^{T}\right] \\
& =(1-p) \sum_{i=0}^{\mathrm{N}-1} p^{i} E\left[\left(\mathbf{x}_{k}-\hat{\mathbf{x}}_{k \mid k-1}\right)\left(\mathbf{z}_{k-i}-\hat{\mathbf{z}}_{k-i \mid k-1}\right)^{T}\right]
\end{aligned}
$$$$
\text { or, } E\left[\left(\mathbf{x}_{k}-\hat{\mathbf{x}}_{k \mid k-1}\right)\left(\mathbf{M}_{1}\right)^{T}\right]=(1-p) \sum_{i=0}^{\mathrm{N}-1} p^{i} \mathbf{P}_{k-i \mid k-1}^{\mathbf{x z}} .
$$

Similarly replacing $\mathbf{M}_{2}$, we get

$$
\begin{aligned}
& E\left[\left(\mathbf{x}_{k}-\hat{\mathbf{x}}_{k \mid k-1}\right)\left(\mathbf{M}_{2}\right)^{T}\right]=E\left[( \mathbf { x } _ { k } - \hat { \mathbf { x } } _ { k | k - 1 } ) \left(\sum_{i=0}^{\mathrm{N}-1}\left(\beta^{(j, i)}-(1-p) p^{i}\right)\right.\right. \\
& \left.\left.\hat{\mathbf{z}}_{k-i \mid k-1}\right)^{T}\right]=\sum_{i=0}^{\mathrm{N}-1} E\left[\left(\beta^{(j, i)}-(1-p) p^{i}\right)\left(\left(\mathbf{x}_{k}-\hat{\mathbf{x}}_{k \mid k-1}\right) \hat{\mathbf{z}}_{k-i \mid k-1}\right)^{T}\right] \\
& =\sum_{i=0}^{\mathrm{N}-1} E\left[\left((1-p) p^{i}-(1-p) p^{i}\right)\left(\left(\mathbf{x}_{k}-\hat{\mathbf{x}}_{k \mid k-1}\right) \hat{\mathbf{z}}_{k-i \mid k-1}\right)^{T}\right]
\end{aligned}
$$$$
\text { or, } \quad E\left[\left(\mathbf{x}_{k}-\hat{\mathbf{x}}_{k \mid k-1}\right)\left(\mathbf{M}_{2}\right)^{T}\right]=0 \text {. }
$$

Substituting (24) and (25) into (23), we get

$$
\mathbf{P}_{k \mid k-1}^{\mathbf{x Y}}=(1-p) \sum_{i=0}^{\mathrm{N}-1} p^{i} \mathbf{P}_{k-i \mid k-1}^{\mathbf{x z}} .
$$

Under the consideration that $\bar{p}_{k}=p^{\mathrm{N}}$ is negligible i.e. no measurement is received, the expressions of $\hat{\mathbf{z}}_{k}, \mathbf{P}_{k \mid k-1}^{\mathbf{z z}}$ and $\mathbf{P}_{k \mid k-1}^{\mathbf{X Z}}$, appeared in the traditional Bayesian approximation framework can be replaced by $\hat{\mathbf{Y}}_{k}, \mathbf{P}_{k \mid k-1}^{\mathbf{Y Y}}$ and $\mathbf{P}_{k \mid k-1}^{\mathbf{x Y}}$ respectively to modify it for dealing with the randomly delayed measurements.

\section{B. Step 2: Incorporating the possibility that a few measure- ments are lost for $(N-1)$-delay}

The probability that no measurement arrives at any time instant $k$ is $\bar{p}_{k}=p^{\mathrm{N}}$. In case when no measurement arrives, the previous measurement is propagated i.e. $\mathbf{y}_{k}=\mathbf{y}_{k-1}$. Now, the expressions of $\hat{\mathbf{Y}}_{k}, \mathbf{P}_{k \mid k-1}^{\mathbf{Y Y}}$ and $\mathbf{P}_{k \mid k-1}^{\mathbf{x Y}}$, derived in the previous step are reformulated and $\hat{\mathbf{y}}_{k}, \mathbf{P}_{k \mid k-1}^{\mathbf{y y}}$ and $\mathbf{P}_{k \mid k-1}^{\mathbf{x y}}$ are derived to get a generalized solution.

Lemma 6: For an $(\mathrm{N}-1)$-delay system, the estimate of measurement can be given as

$$
\hat{\mathbf{y}}_{k \mid k-1}=(1-p) \sum_{i=0}^{\mathrm{N}-1} p^{i} \hat{\mathbf{z}}_{k-i \mid k-1}+p^{\mathrm{N}} \hat{\mathbf{y}}_{k-1 \mid k-2} .
$$

Proof:

$$
\mathbf{y}_{k}=\sum_{i=0}^{\mathrm{N}-1} \beta^{(j, i)} \mathbf{z}_{k-i}+\underbrace{\left(1-\sum_{i=0}^{\mathrm{N}-1} \beta^{(j, i)}\right) \mathbf{y}_{k-1}}_{\mathbf{M}_{3}}=\mathbf{Y}_{k}+\mathbf{M}_{3} .
$$

hence, $\hat{\mathbf{y}}_{k \mid k-1}=\hat{\mathbf{Y}}_{k \mid k-1}+E\left[\left(1-\sum_{i=0}^{N-1} \beta^{(j, i)}\right)\right] \hat{\mathbf{y}}_{k-1 \mid k-2}$
Substituting (12) and (10)

$$
\hat{\mathbf{y}}_{k \mid k-1}=(1-p) \sum_{i=0}^{\mathrm{N}-1} p^{i} \hat{\mathbf{z}}_{k-i \mid k-1}+p^{\mathrm{N}} \hat{\mathbf{y}}_{k-1 \mid k-2} .
$$

Before proceeding forward, we make an assumption that $\mathbf{P}_{k \mid k-1}^{\mathbf{y y}} \approx \mathbf{P}_{k \mid k-1}^{\mathbf{Y Y}}+E\left[\left(\mathbf{M}_{3}-E\left[\mathbf{M}_{3}\right]\right)\left(\mathbf{M}_{3}-E\left[\mathbf{M}_{3}\right]\right)^{T}\right]$. This assumption is useful for deriving the measurement covariance.

Remark 2: The above assumption does not affect the performance much as the estimates of cross terms cancel each other due to the independence of $\mathbf{z}_{k}$ and $\mathbf{z}_{j}(\forall j \neq k)$. The cross terms are non-zero only when $\mathbf{y}_{k}$ and $\mathbf{y}_{k-1}$ both receives the same measurement $\mathbf{z}_{d}$ where $d<k$ and the probability of this occurrence is negligible, as the probability of occurrence of $\mathbf{M}_{3}$ is already quite small in practice.

Now, Under the assumption made on $\mathbf{M}_{3}$ earlier, we may write

$$
\begin{aligned}
\mathbf{P}_{k \mid k-1}^{\mathbf{y y}} \approx & \mathbf{P}_{k \mid k-1}^{\mathbf{Y Y}}+E\left[\left(\mathbf{M}_{3}-E\left[\mathbf{M}_{3}\right]\right)\left(\mathbf{M}_{3}-E\left[\mathbf{M}_{3}\right]\right)^{T}\right] \\
& =\mathbf{P}_{k \mid k-1}^{\mathbf{Y Y}}+E\left[\left(\left(1-\sum_{i=0}^{\mathrm{N}-1} \beta^{(j, i)}\right) \mathbf{y}_{k-1}-p^{\mathrm{N}} \hat{\mathbf{y}}_{k-1 \mid k-2}\right)\right. \\
& \left.\left(\left(1-\sum_{i=0}^{\mathrm{N}-1} \beta^{(j, i)}\right) \mathbf{y}_{k-1}-p^{\mathrm{N}} \hat{\mathbf{y}}_{k-1 \mid k-2}\right)^{T}\right] .
\end{aligned}
$$

Further, $E\left[\left(1-\sum_{i=0}^{\mathrm{N}-1} \beta^{(j, i)}\right)^{2}\right]=E\left[\left(1-\sum_{i=0}^{\mathrm{N}-1} \beta^{(j, i)}\right)\right]=p^{\mathrm{N}}$.

Also, $p^{2 \mathrm{~N}} \approx p^{\mathrm{N}}$ for small $p$ or higher $\mathrm{N}$.

hence, $\mathbf{P}_{k \mid k-1}^{\mathbf{y y}} \approx \mathbf{P}_{k \mid k-1}^{\mathbf{Y Y}}+p^{\mathrm{N}} E\left[\left(\mathbf{y}_{k-1}-\hat{\mathbf{y}}_{k-1 \mid k-2}\right)\left(\mathbf{y}_{k-1}-\hat{\mathbf{y}}_{k-1 \mid k-2}\right)^{T}\right]$.

Replacing (13), we get

$$
\begin{aligned}
& \mathbf{P}_{k \mid k-1}^{\mathbf{y y}} \approx(1-p) \sum_{i=0}^{\mathrm{N}-1} p^{i} \mathbf{P}_{k-i \mid k-1}^{\mathbf{z z}}+(1-p) \sum_{i=0}^{\mathrm{N}-1} p^{i}(1- \\
& \left.p^{i}(1-p)\right)\left(\left(\hat{\mathbf{z}}_{k-i \mid k-1}\right)\left(\hat{\mathbf{z}}_{k-i \mid k-1}\right)^{T}\right)+p^{\mathrm{N}} \mathbf{P}_{k-1 \mid k-2}^{\mathbf{y y}} .
\end{aligned}
$$

Lemma 7: For an ( $\mathrm{N}-1)$-delay system, the cross-covariance between the state and measurement could be given as

$$
\mathbf{P}_{k \mid k-1}^{\mathbf{x y}}=(1-p) \sum_{i=0}^{\mathrm{N}-1} p^{i} \mathbf{P}_{k-i \mid k-1}^{\mathbf{x z}}+p^{\mathrm{N}} \mathbf{P}_{k-1 \mid k-2}^{\mathbf{x y}} .
$$

Proof: We may write,

$\mathbf{P}_{k \mid k-1}^{\mathbf{x y}}=E\left[\left(\mathbf{x}_{k}-\hat{\mathbf{x}}_{k \mid k-1}\right)\left(\mathbf{Y}_{k}-\hat{\mathbf{Y}}_{k \mid k-1}\right]\right)^{T}+E\left[\left(\mathbf{x}_{k}-\hat{\mathbf{x}}_{k \mid k-1}\right)\right.$

$\left.\left(\mathbf{M}_{3}-E\left[\mathbf{M}_{3}\right]\right)^{T}\right]=\mathbf{P}_{k \mid k-1}^{\mathbf{x Y}}+E\left[\left(\mathbf{x}_{k}-\hat{\mathbf{x}}_{k \mid k-1}\right)\left(\left(1-\sum_{i=0}^{\mathrm{N}-1} \beta^{(j, i)}\right)\right.\right.$ $\left.\left.\mathbf{y}_{k-1}-p^{\mathrm{N}} \hat{\mathbf{y}}_{k-1 \mid k-2}\right)^{T}\right]=\mathbf{P}_{k \mid k-1}^{\mathbf{x Y}}+p^{\mathrm{N}} \mathbf{P}_{k-1 \mid k-2}^{\mathbf{x y}}$.

Replacing (22), we get

$$
\mathbf{P}_{k \mid k-1}^{\mathbf{x y}}=(1-p) \sum_{i=0}^{\mathrm{N}-1} p^{i} \mathbf{P}_{k-i \mid k-1}^{\mathbf{x z}}+p^{\mathrm{N}} \mathbf{P}_{k-1 \mid k-2}^{\mathbf{x y}} .
$$


Remark 3: The conventional Bayesian approximation framework for filtering using numerical integration could be modified to deal with the problem of randomly delayed measurements by replacing the conventional expressions of estimates and covariances of measurement by $\hat{\mathbf{y}}_{k}, \mathbf{P}_{k \mid k-1}^{\mathbf{y y}}$ and $\mathbf{P}_{k \mid k-1}^{\mathbf{x y}}$ respectively and keeping all the other expressions as it is.

In step 2 (section II-B), $\hat{\mathbf{x}}_{k+1 \mid k+1}$ is computed using $y_{k}$ if the measurement received at $k^{t h}$ instant is delayed by more than $(\mathrm{N}-1)$ steps. It may result in poor accuracy for some problems (especially, if the sampling interval is high). For such problems, $\mathrm{N}$ must be chosen sufficiently high in order to minimize/circumvent the possibilities of lost measurements. Alternatively, prediction could be performed without measurement update at those steps.

\section{Simulation Results}

In this section, for comparison purpose, the proposed method is implemented to solve two different filtering problems where delay in measurement is likely.

\section{A. Problem 1}

The process and measurement models for an arbitrary dimensional system are [15] $\mathbf{x}_{k}=2 \cos \left(\mathbf{x}_{k-1}\right)+$ $\mathbf{q}_{k-1}$, and $\quad \mathbf{y}_{k}=\sqrt{1+\mathbf{x}_{k}^{T} \mathbf{x}_{k}}+\mathbf{v}_{k}$. The noise covariances are $\mathbf{Q}=5 \mathbf{I}_{n}$ and $\mathbf{R}=5$. The filter is initialized with $\mathbf{x}_{0}=0.1_{n \times 1}, \hat{\mathbf{x}}_{0}=15_{n \times 1}$ and $\mathbf{P}_{0 \mid 0}=5 \mathbf{I}_{n}$. The states are estimated upto 200 time steps and the results are ensured with 200 independent Monte Carlo runs by considering $n=6$.

For filtering, the CQKF is applied with $2^{\text {nd }}$ order GaussLaguerre quadrature rule. The simulation is performed considering one and two time step delays in measurement. The results are compared in terms of the root mean square error (RMSE) averaged along the time step. The average RMSE against the different probability $p=P\left(\beta_{j}=1\right)$ i.e. $0.1 \leq$ $p \leq 0.9$ are plotted in figure- 1 and figure- 2 for one delay case and two delay case respectively. The subfigures (a) to (f) are corresponding plots for state- 1 to state- 6 of the sixth dimensional system.

The simulation is performed with the conventional CQKF and the modified $\mathrm{CQKF}$ as proposed in this paper, represented by CQKF-RD. The conventional CQKF suffers significant numerical errors in matrix inversion for higher probability of delay $(p)$ and hence its square-root version [16] is used for implementation. From the plots, it could be concluded that the performance of CQKF-RD is significantly better than the ordinary CQKF, in the presence of a delay.

\section{B. Problem 2}

In this problem, we estimate the amplitude and the frequency of multiple superimposed sinusoids [17], [18]. The delay in measurement is probable due to the bandwidth criteria. In this paper, the number of sinusoids are considered to be three, hence the state variable can be represented as $\mathbf{x}=\left[f_{1}, f_{2}, f_{3}, a_{1}, a_{2}, a_{3}\right]^{T}$, where $f_{i}$ and $a_{i}$ are the frequency and amplitude of the $i^{\text {th }}$ sinusoid. The process model follows random walk model, i.e.

$$
\mathbf{x}_{k}=\mathbf{I} \mathbf{x}_{k-1}+\mathbf{q}_{k},
$$

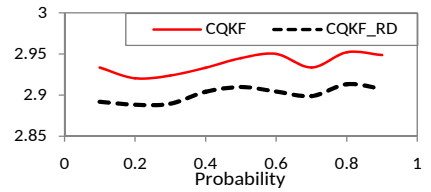

(a)

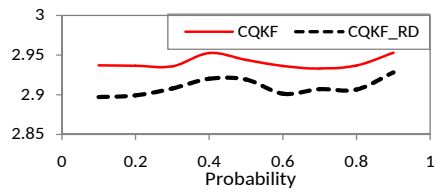

(c)

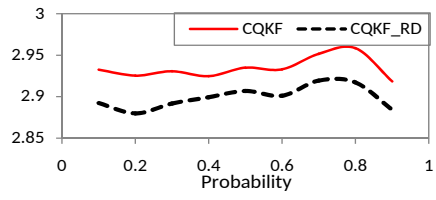

(e)

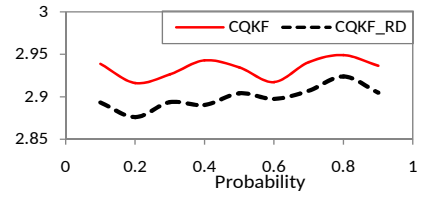

(b)

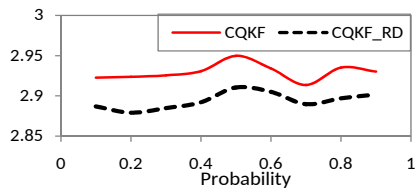

(d)

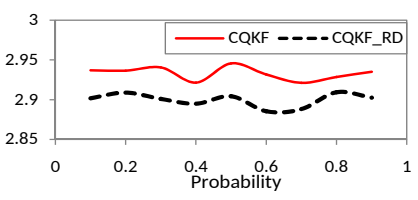

(f)
Fig. 1. Average RMSE against probability plot for one delay, for (a) state-1, (b) state-2, (c) state-3, (d) state-4, (e) state-5 (f) state-6.

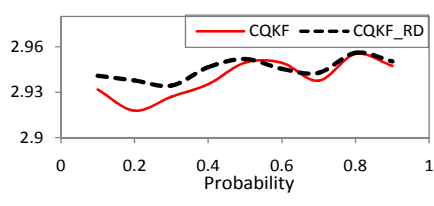

(a)

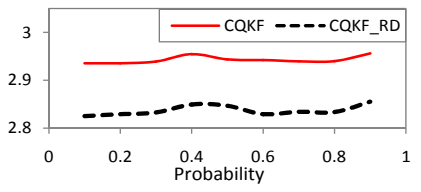

(c)

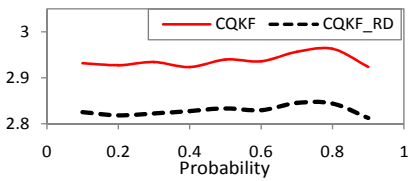

(e)

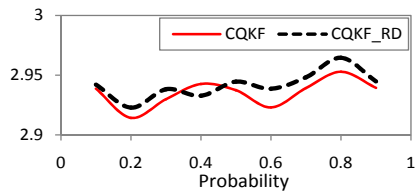

(b)

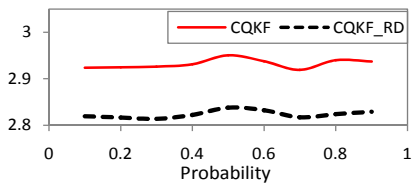

(d)

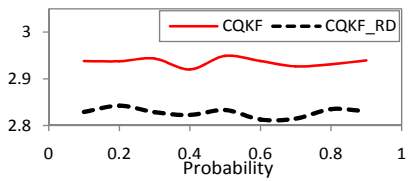

Fig. 2. Average RMSE against probability plot for two delays, for (a) state-1, (b) state-2, (c) state-3, (d) state-4, (e) state-5 (f) state-6.

The measurement equation is [19]

$\mathbf{y}_{k}=\left[\sum_{j=1}^{3} a_{j, k} \cos \left(2 \pi f_{j, k} k T\right) \sum_{j=1}^{3} a_{j, k} \sin \left(2 \pi f_{j, k} k T\right)\right]^{T}+\mathbf{v}_{k}$,

where $T$ is the sampling time considered as $0.1667 \mathrm{~ms}$.

The noise covariances are $\mathbf{Q}=\operatorname{diag}\left(\left[\begin{array}{llll}\sigma_{f}^{2} & \sigma_{f}^{2} & \sigma_{f}^{2} & \sigma_{a}^{2}\end{array}\right.\right.$ $\left.\left.\sigma_{a}^{2} \sigma_{a}^{2}\right]\right)$ i.e. $\sigma_{f}=\sqrt{2.55} \mathrm{mHz}$ and $\sigma_{a}=2 m V$, and $\mathbf{R}=\operatorname{diag}\left(\left[\begin{array}{ll}0.009 V^{2} & 0.009 V^{2}\end{array}\right]\right)$. The initial true values and estimates are considered as [20 $\left.\begin{array}{llllll}200 & 1000 & 2000 & 2 & 2 & 2\end{array}\right]^{T}$ and

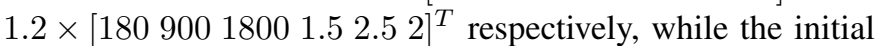
error covariance is taken as $\operatorname{diag}\left(\left[\begin{array}{llllll}20^{2} & 20^{2} & 20^{2} & 0.5 & 0.5 & 0.5\end{array}\right]\right)$. The states are estimated for 750 steps and the results are ensured with 500 Monte Carlo runs. The results are compared in terms of the RMSE. 


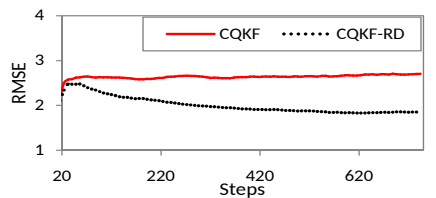

(a)

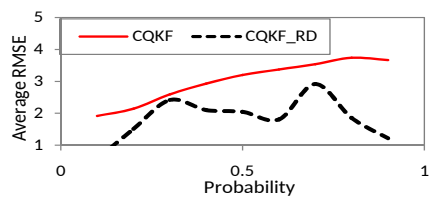

(c)

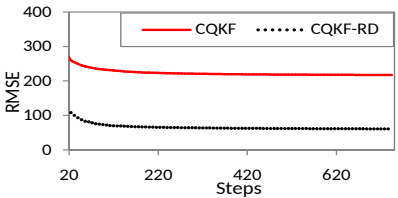

(b)

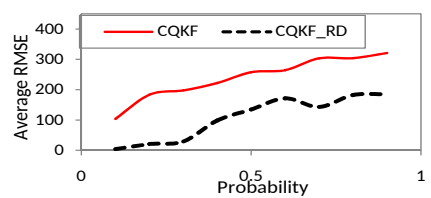

(d)
Fig. 3. One delay: (a) RMSE (in $V$ ) vs time step plot for amplitude considering $p=0.3$ (b) RMSE (in Hz) vs time step plot for frequency considering $p=0.3$ (c) Average RMSE vs probability plot for amplitude (c) Average RMSE vs probability plot for frequency.

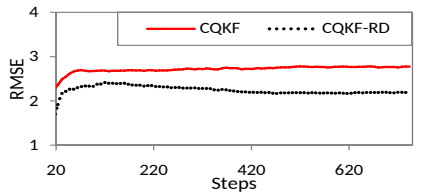

(a)

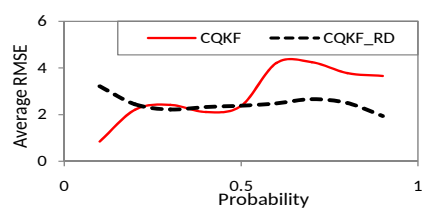

(c)

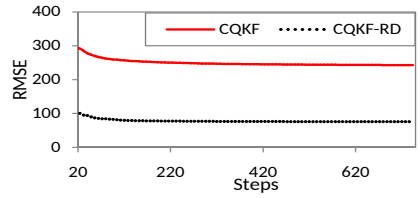

(b)

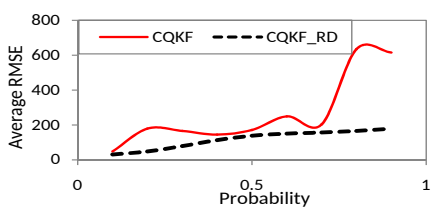

(d)

Fig. 4. Two delays: (a) RMSE (in $V$ ) vs time step plot for amplitude considering $p=0.3$ (b) RMSE (in Hz) vs time step plot for frequency considering $p=0.3$ (c) Average RMSE vs probability plot for amplitude (c) Average RMSE vs probability plot for frequency.

Figures 3(a) and 3(b) represent the RMSE plot of frequency and amplitude respectively, while Figures 3(c) and 3(d) show the average RMSE plotted against the probability for frequency and amplitude for the case of one delay in measurement. Similarly, Figures 4(a) to 4(d) represent the same plots for two delay case. For this problem as well, the RMSEs are compared for the ordinary CQKF and CQKF-RD. During simulation, the square-root version is used for ordinary $\mathrm{CQKF}$ as it suffers with numerical errors in matrix inversion. It is found that the result is improved for CQKF-RD.

For one delay case, the authors also compared the new algorithm with the algorithm in [19]. It was found that the performance of the two algorithms was comparable at the low end of probabilities, although our algorithm outperformed at large delay probabilities. Details are omitted for brevity.

\section{Discussions AND CONCLUSIONS}

In recent years, the conventional Bayesian approach of filtering has been most accepted one. This approach works only if the measurement is available at every time instant. Hence, its practical applicability vanishes if the measurement reaches to the filter after some delay in time. The delayed measurement problem is very common in maneuvering and underwater target tracking problems where distance and sensor response time matters and in the filtering and estimation problems occurring in communication systems where bandwidth matters.

In this paper, the Bayesian approximation framework for filtering using numerical integration is enhanced for the problems where the measurement is expected to arrive after some delay. To compare the performance, two nonlinear filtering problems are solved using the conventional CQKF and modified CQKF for randomly delayed measurements (CQKF-RD). It is found that the RMSE is lower for CQKF-RD which concludes that the proposed modification can be accepted for real life applications if delay in measurement is probable.

\section{ACKNOWLEDGEMENT}

The research of Dr P. Date was partially supported by EPSRC, UK, under grant reference EP/L019477/1.

\section{REFERENCES}

[1] M. S. Arulampalam, S. Maskell, N. Gordon and T. Clapp, "A tutorial on particle filters for online nonlinear/non-Gaussian Bayesian tracking," IEEE Trans. Signal Process., vol. 50, no. 2, pp.174-188, Feb. 2002.

[2] Y. Bar-Shalom, X. R. Li and T. Kirubarajan, Estimation with application to tracking and navigation, Wiley-Interscience Pub., New York, 2001.

[3] J. Simon, J. Uhlmann, F. Hugh and W. Durrant, "A new method for the nonlinear transformation of means and covariances in filters and estimators," IEEE Trans. Autom. Control, vol. 45, no. 3, pp. 477-482, Mar. 2000.

[4] I. Arasaratnam, S. Haykin and R. J. Elliott, "Discrete-time nonlinear filtering algorithms using Gauss-Hermite quadrature," Proc. IEEE, vol. 95, no. 5, pp. 953-977, May 2007.

[5] K. Ito and K. Xiong, "Gaussian filters for nonlinear filtering problems," IEEE Trans. Autom. Control, vol. 45, no. 5, pp. 910-927, May 2000.

[6] I. Arasaratnam and S. Haykin, "Cubature kalman filter," IEEE Trans. Autom. Control, vol. 54, no. 6, pp. 1254-1269, May 2009.

[7] S. Bhaumik and Swati, "Cubature quadrature Kalman filter," IET Signal Processing, vol. 7, no. 7, pp. 1-9, Sep. 2013.

[8] R. L. Moose and T. Dailey, "Adaptive underwater target tracking using passive multipath time-delay measurements," IEEE Trans. Acoust. Speech Signal Process., vol. 33, no. 4, pp. 778-787 , Aug. 1985.

[9] J. Nilsson, B. Bernhardsson and B. Wittenmark, "Stochastic analysis and control of real-time systems with random time delays," Automatica, vol. 34, no. 1, pp. 57-64, Jan. 1998.

[10] I. V. Kolmanovskya and T. L. Maizenbergb, "Optimal control of continuous-time linear systems with a time-varying, random delay," Systems Control Letters, vol. 44, no. 2, pp. 119-126, Oct. 2001.

[11] A. H. Carazo and J. L. Perez, "Extended and unscented filtering algorithms using one-step randomly delayed observations," Appl. Maths. Comp.,vol. 190, no. 2, pp. 1375-1393, Jul. 2007.

[12] A. H. Carazo and J. L. Perez, "Unscented filtering algorithm using twostep randomly delayed observations in nonlinear systems," Appl. Maths. Modelling, vol. 33, no. 9, pp. 3705-3717, Sep. 2009.

[13] X. Wang, Y. Liang, Q. Pan and C. Zhao, "Gaussian filter for nonlinear systems with one-step randomly delayed measurements," Automatica, vol. 49, no. 4, pp. 976-986, April 2013.

[14] A. H. Carazo and J. L. Perez, "Unscented filtering from delayed observations with correlated noises," Math. Prob. Eng., vol. 2009, pp. 1-9, Jun. 2009.

[15] L. Chang, B. Hu, A. Li and F. Qin, "Transformed unscented Kalman filter,' IEEE Trans. Autom. Cont., vol. 58, no. 1, pp. 252-257, Dec. 2012.

[16] S. Bhaumik, and Swati, "Square-root cubature quadrature Kalman filter," Asian J. control, vol. 16, no. 2, pp. 617-622, Mar. 2014.

[17] M. Niedzwiecki and P. Kaczmarek, "Estimation and tracking of complex-valued quasi-periodically varying systems," Automatica, vol. 41, no. 9, pp. 1503-1516, Sep. 2005.

[18] J. Reddy, P. K. Dash, R. Samantaray and A.K. Moharana, "Fast tracking of power quality disturbance signals using an optimized unscented filter," IEEE Trans. Instrum. Meas., vol. 58, no. 12, pp. 3943-3952, Dec. 2009.

[19] P. Closas, C. F. Prades and J. V. Valls, "multiple quadrature Kalman filtering," IEEE Trans. signal process., vol. 60, no. 12, pp. 6125-6137, Sep. 2012 